\title{
FLI-06 Intercepts Notch Signaling And Suppresses The Proliferation And Self-renewal Of Tongue Cancer Cells
}

This article was published in the following Dove Press journal: OncoTargets and Therapy

\begin{abstract}
Rui-huan Gan, ',* Li-song Lin, (iD) 2 ,* Jing Xie, iD ${ }^{3-5, *}$ Li Huang, ${ }^{2,4}$ Lin-can Ding, ${ }^{2}$ Bo-hua Su, ${ }^{2}$ Xian-e Peng, ${ }^{1,5}$ Da-li Zheng, (iD) ${ }^{4,5}$ You-guang Lu ${ }^{1,3}$

'Department of Epidemiology and Health Statistics, Fujian Provincial Key Laboratory of Environment Factors and Cancer, School of Public Health, Fujian Medical University, Fuzhou 350I22, People's Republic of China; ${ }^{2}$ Department of Oral and Maxillofacial Surgery, Affiliated First Hospital of Fujian Medical University, Fuzhou 350005, People's Republic of China; ${ }^{3}$ Department of Preventive Dentistry, School and Hospital of Stomatology, Fujian Medical University, Fuzhou 350000, People's Republic of China; ${ }^{4}$ Key Laboratory of Stomatology of Fujian Province, School and Hospital of Stomatology, Fujian Medical University, Fuzhou 350004, People's Republic of China; ${ }^{5}$ Key Laboratory of Ministry of Education for Gastrointestinal Cancer, Fujian Medical University, Fuzhou 350I22, People's Republic of China

*These authors contributed equally to this work
\end{abstract}

Correspondence: You-guang Lu Department of Preventive Dentistry, School and Hospital of Stomatology, Fujian Medical University, 246 Yang Qiao Middle Road, Fuzhou 350000, People's

Republic of China

Tel +86591 83736429

Fax +86 591 83720599

Email fjlyg63@fjmu.edu.cn

Xian-e Peng

Department of Epidemiology and Health Statistics, Fujian Provincial Key Laboratory of Environment Factors and Cancer, School of Public Health, Fujian Medical University, I Xue Yuan Road,

University Town, Fuzhou 350 I22, People's Republic of China

Tel +86 I 38 095। 6580

Fax +8659183720599

Email fmuxe@163.com
Purpose: The Notch signaling pathway plays an oncogenic role in tongue squamous cell carcinoma. The aim of this study was to inhibit the proliferation and self-renewal of tongue cancer cells by applying Notch signaling pathway inhibitor FLI-06 (Selleck, USA) and to lay a foundation for the clinically targeted treatment of tongue cancer for the future.

Methods: The mRNA expression level of Notch1 and the overall survival rate of patients with tongue cancer were examined by analyzing the TCGA database. Tongue cancer cells were treated with FLI-06. Cell proliferation, apoptosis, and stem cell self-renewal ability were tested in appropriate ways. A xenograft mouse model was established to observe tumor growth.

Results: From the TCGA data, we demonstrated that patients with high expression of Notch1 had a poor prognosis. We observed that the Notch signaling pathway inhibitor FLI-06 can restrain the activation of the Notch signaling pathway, decrease cell proliferation and induce cell apoptosis in vitro. The xenograft experiment indicated that intraperitoneal injection of FLI-06 inhibited tumor growth and increased cell apoptosis. FLI-06 suppressed both the mRNA and protein expression of Notch receptor and Notch targeted genes. We also observed that FLI-06 suppressed the proliferation of tongue cancer stem cells.

Conclusion: FLI-06 can block the proliferation and self-renewal of tongue cancer cells. It is inferred that this compound, which inhibits the Notch signaling pathway, may serve as a potential targeted drug for the treatment of tongue cancer in the clinic.

Keywords: tongue cancer, Notch signaling pathway, Notch inhibitor, cancer stem cells

\section{Introduction}

Oral cancer is a common cancer that could significantly impact patients' quality of life, but it is often ignored by the public. Based on GLOBOCAN 2018 (http://gco. iarc.fr/today/home), we know that the incidence and mortality of lip and oral cavity cancer ranks 18 th worldwide. Despite rapid developments in medicine, the incidence and mortality of oral cancer has not taken a turn for the better. On the basis of data on the incidence and mortality of oral and oropharyngeal cancers in China, Zhang and colleagues ${ }^{1}$ estimated that the 5-year crude incidence would show a rising trend in the next two decades in China. Notably, much of the research on oral cancer has reported that patients with oral cancers are becoming younger and the number of female patients is increasing. ${ }^{2,3}$ In addition to common etiologies, such as smoking, HPV infection, aging and so on, there are some unknown but significant etiologies, for instance, molecular biological etiologies, that need to be uncovered. Tongue cancer is one of the most common cancers in the oral cavity, 
and the etiology of tongue cancer, especially the molecular mechanism, remains unclear. It is crucial to find some etiology at the molecular level to improve the prognosis of tongue cancer patients.

The Notch signaling cascade is an evolutionarily conserved and ubiquitous pathway that was discovered more than 100 years ago in the fruit fly Drosophila with notch wings. ${ }^{4}$ The Notch signaling pathway consists of four receptors and five ligands and plays an important role in development, tissue homeostasis, and disease in mammals. ${ }^{5}$ The canonical Notch signaling pathway involves cell-to-cell surface signaling, whereby cells with Notch receptors are activated after coming into contact with cells with Notch ligands. ${ }^{6,7}$ The Notch receptor is cleaved by gamma secretase in the third cleavage $(\mathrm{S} 3)^{8}{ }^{8}$ The $\mathrm{S} 3$ is regulated by a presenilin-dependent $\gamma$-secretase protease complex, consists by presenilin 1 (PSEN1) or PSEN2, nicastrin, presenilin enhancer 2 (PEN2) and anterior pharynxdefective1 (APH1). ${ }^{8,9}$ After the gamma secretase proteolysis Notch receptor, Notch intracellular domain (NICD) would be released from the membrane to the cytoplasm. Following the NICD translocation and biding to CSL, the pathway is activated. ${ }^{10}$ The Notch signaling pathway may play dual roles in different cancers. Much research on malignancies, such as Tcell acute lymphoblastic leukemia, ${ }^{11}$ bladder cancer, ${ }^{12}$ and prostate cancer, ${ }^{13}$ has reported that the Notch signaling pathway is oncogenic and could promote cancer progression and metastasis. However, some studies have also stated that the Notch signaling pathway acts as a tumor suppressor, such as in forebrain glioma, ${ }^{14}$ cutaneous SCC, ${ }^{15}$ and colorectal cancer. ${ }^{16}$ Our previous research emphasized that Notch1 acts as an oncogene in tongue cancer and could promote tongue cancer cell proliferation and migration and inhibit cell apoptosis. ${ }^{17}$ The aim of this study was to discover a new approach to target the Notch signaling pathway and achieve the possibility of a targeted treatment for tongue cancer.

The b-annulated dihydropyridine FLI-06 is a novel small molecular chemical compound, which is described could inhibit general secretion at a step before exit from the endoplasmic reticulum. ${ }^{18}$ Therefore, FLI-06 could inhibit Notch protein through the early secretory pathway. ${ }^{19,20}$ Some colleague had reported that FLI-06 could suppresses the progressive of esophageal squamous cell carcinoma. ${ }^{21}$ But there are still not studies about FLI-06 affect tongue carcinoma squamous cell.
Since FLI-06 as a novel Notch inhibitor, we focus on it and want to find the effect of it on cell proliferation, apoptosis, cell cycle and self-renew ability of tongue cancer cells.

\section{Materials and methods Cell Lines And Cell Cultures}

CAL-27 cell lines were bought from the ATCC (American Type Culture Collection, USA), and TCA-8113 cell lines were a gift from Dr. Chen (College of Stomatology, Ninth People's Hospital, Shanghai Jiao Tong University School of Medicine). CAL-27 cell lines were grown in DMEM/ HIGH GLUCOSE (HyClone, Catalog \# SH30022.01) supplemented with 10\% FBS, and TCA-8113 cell lines were cultured in RPMI medium modified (HyClone, Catalog \# SH30809.01) supplemented with 10\% FBS. Both cell lines were incubated at $37^{\circ} \mathrm{C}$ in a humidified atmosphere of 95\% air with $5 \% \mathrm{CO}_{2}$. All cell lines were STR (Short Tandem Repeat)authenticated annually by Shanghai Biowing Applied Biotechnology Co. LTD., Shanghai, China.

\section{TCGA Datamining}

The mRNA expression data and clinical information from the HNSC dataset were downloaded from the TCGA data portal (http://gdc.cancer.gov). The dataset was obtained on July 28, 2018, which included 501 HNSC samples and 41 normal tissues. Among these samples, there are 149 tongue cancer samples and 15 normal tongue tissues. The mRNA expression level was log2-transformed to calculate the correlation and fold change with the FPKM value plus 0.01 (to avoid error during $\log 2$ transformation) and the patients were divided to Notch1-High $(n=74)$ and Notch1Low $(n=75)$ from the median according to Notch1 expression level.

\section{Cell Proliferation Assay}

Cell proliferation was detected by Cell Counting Kit-8 (CCK8) reagent and colony formation assays as previously described. $^{22}$

\section{Apoptosis Assay}

Apoptotic cells were detected using a FITC Annexin V Apoptosis Detection Kit (BD Pharmingen, Catalog \# 556547) followed by flow cytometry analysis. 


\section{TUNEL Assay}

We then attached CAL-27 cells to a microscope slide following the manufacturer's instructions (PROMEGA, Catalog \# G3250). We immediately analyzed the samples under a fluorescence microscope using a standard fluorescein filter set to view the green fluorescence of fluorescein at $520 \pm 20 \mathrm{~nm}$ and to view the red fluorescence of propidium iodide at $>620 \mathrm{~nm}$. We took pictures of five random fields (at $400 \times$ ).

\section{Cell Cycle Test}

We trypsinization and collected CAL-27 cells after treatment with the inhibitor for $48 \mathrm{~h}$, soaked and fixed the cells with $70 \%$ ethanol at $4^{\circ} \mathrm{C}$ overnight, and then, after low-speed centrifugation, the cells were collected after washing with PBS, and $400 \mu \mathrm{l} \mathrm{PI/RNase} \mathrm{Staining} \mathrm{Buffer} \mathrm{(BD} \mathrm{Pharmingen,}$ Catalog \# 554655) was added. The percentage of each phase of cells was quantified using a BD FACS Verse flow cytometer.

\section{Establishing A Xenograft Tumor Model}

Female BALB/c nude mice (6-8 weeks of age) were purchased from the Center for Animal Experiments of Fujian Medical University. Prior to injection, 15 nude mice were assigned at random to three groups with five mice per group. Cells $\left(2 \times 10^{6}\right)$ were suspended in $0.2 \mathrm{~mL}$ serum-free DMEM and injected into the right axillary fossa of each mouse. Tumor size was calculated using the formula $\mathrm{V}=$ width $^{2} \times$ length $/ 2$. At the end of the experiment, the tumors were harvested and weighed. All animal experiments were performed according to the guidelines for the care and use of laboratory animals and the protocol was approved by the biomedical ethics research committee of Fujian Medical University.

\section{Quantitative Real-time PCR}

The expression of Notch-relevant mRNAs was tested by quantitative real-time PCR as previously described. ${ }^{22}$ The sequences of the primers are listed in Table 1. Data were analyzed according to the $2^{-\Delta \Delta C t}$ method.

\section{Western Blotting}

The expression of Notch-relevant proteins was tested by Western blotting as previously described. ${ }^{22}$ The primary and secondary antibody dilutions are shown in Table 2.

\section{Cancer Stem Cell Sphere Culture}

Tongue cancer cells were collected and serum was removed. Then, CAL-27 and TCA- 8113 cells were suspended in serum-free DMEM/F12 (GIBCO, Catalog \# 12400-024) and stem cell medium (STEMCELL

Table I Real-time PCR Primers

\begin{tabular}{|l|l|l|}
\hline GENE & FORWARD & REVERSE \\
\hline Notchl & GGAAGTTGAACGAGCATAGTCC & GCATGATGCCTACATTTCAAGA \\
Notch2 & TATGTTCAAGTGCAGGAATTGG & AGAGTCGGGAATTCACCTGTTA \\
HESI & AGGCGGACATTCTGGAAATG & CGGTACTTCCCCAGCACACTT \\
HEYI & CGAGGTGGAGAAGGAGAGTG & CTGGGTACCAGCCTTCTCAG \\
HEY2 & GAACAATTACTCGGGGCAAA & TCAAAAGCAGTTGGCACAAG \\
ALDHI & GATCCAGGGCCGTACAATACCA & AGTGCAGGCCCTATCTTCCAAA \\
SOX2 & ATGGACAGTTACGCGCACAT & CGAGCTGGTCATGGAGTTGT \\
OCT4 & GCGACTATGCACAACGAGAG & CAGAGTGGTGACGGAGACA \\
SLUG & GAGCATTTGCAGACAGGTCA & CCTCATGTTTGTGCAGGAGA \\
GAPDH & AGGTCAGGGATGACCTTGCC \\
ACTB & TGCACCACCAACTGCTTAGC & TTTTAGGATGGCAAGGGACTTC \\
\hline
\end{tabular}

Table 2 Information About Antibodies

\begin{tabular}{|l|l|l|l|}
\hline ANTIBODY & BRAND & CATALOG NO. & DILUTION MULTIPLE \\
\hline NotchI & Sigma & N4788 & $1: 500$ \\
HESI & Cell Signaling Technology & \# II988S & $1: 500$ \\
HEY2 & Thermo Fisher Scientific & PA5-25807 & $1: 500$ \\
$\beta$-actin & Sigma & A2228 & $1: 1000$ \\
\hline
\end{tabular}


Technologies Inc, Catalog \# 05926), and the two media ratios were 1:1. The cells were subsequently cultured in 96-well plates in ultralow attachment dishes (Corning Life Sciences, Catalog \# 3262) at a density of 200 cells/ $100 \mu 1$ for CAL-27 cells or 100 cells $/ 100 \mu 1$ for TCA8113 cells.

\section{Statistical Analysis}

The statistical analyses of experiments in this study were performed using one-way analysis of variance. In the figures, $\mathrm{P}>0.05$ is represented as not significant (n.s), while $\mathrm{P}<0.05$ was considered statistically significant $(*$ indicates $\mathrm{P}<0.05$ and $* *$ indicates $\mathrm{P}<0.01)$.

\section{Results}

\section{The High Expression Of Notch I Is} Related To A Poor Prognosis In Tongue Carcinoma Patients

As our previous studies reported, the expression of Notch 1 is increased in tongue cancer tissues relative to adjacent normal tissues, and the high expression of Notch 1 is related to a poor differentiation of tongue cancer. ${ }^{17}$ Similarly, we explored the TCGA database from public data and found that the mRNA expression level of Notch1 in tongue cancer was not significantly different from that in normal tissues (Figure 1A). However, according to the database, the high expression of Notch1 may leads to a shorter overall survival of the patients (Figure 1B, $\mathrm{P}=0.06$ ). Although the difference of overall survival of these two groups was not significant, which may be due to the limited number of

A

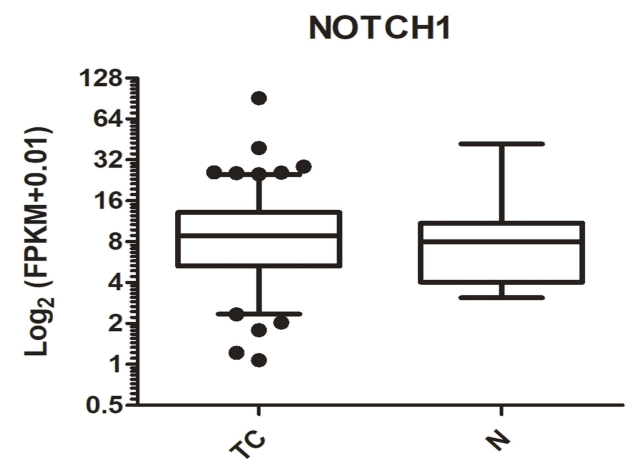

tongue cancer samples in the TCGA database, but the $\mathrm{P}$ value is close to the cutoff, and we found that the trend was consistent with our previous studies. Above all, we know that Notch1 acts as an oncogene in tongue cancer and that the high expression of Notch 1 is closely related with a poor prognosis.

\section{The Notch Signaling Pathway Inhibitor Restrains Tongue Cancer Cell Proliferation In Vitro}

Because of the important role of Notch1 in tongue carcinoma, it is reasonable to identify inhibitors of the Notch signaling pathway to address tongue cancer. Based on the literature search, we chose one kind of Notch signaling inhibitor FLI06 . To test the half maximal inhibitory concentration $\left(\mathrm{IC}_{50}\right)$ of FLI-06, we diluted the inhibitor with semilogarithmic concentrations, added them to tongue cancer cell lines for $48 \mathrm{~h}$ or $72 \mathrm{~h}$, then tested the cell viability by using the CCK 8 assay. We found that FLI-06, a novel small molecule inhibitor, could block tongue cancer cell growth in a concentration-dependent manner. The $\mathrm{IC}_{50}$ of FLI-06 was approximately $4.24 \sim 5.26 \mu \mathrm{M}$ and $2.8 \sim 3.5 \mu \mathrm{M}$ in CAL-27 cells and TCA-8113 cells, respectively (Figure 2A and B). A time-course CCK 8 assay was used to measure the viability of CAL-27 and TCA- 8113 cells treated with FLI-06, and the results showed that FLI-06 suppressed the growth of tongue cancer cells (Figure $2 \mathrm{C}$ and $\mathrm{D}$ ). The long-term proliferation of CAL-27 and TCA-8113 cells was also inhibited by FLI-06, as shown by a colony formation assay (Figure 2E and F). These data indicate that the novel small molecule Notch inhibitor FLI-06 can suppress the proliferation of tongue cancer cells and may serve as an effective drug for the treatment of tongue cancer.

B

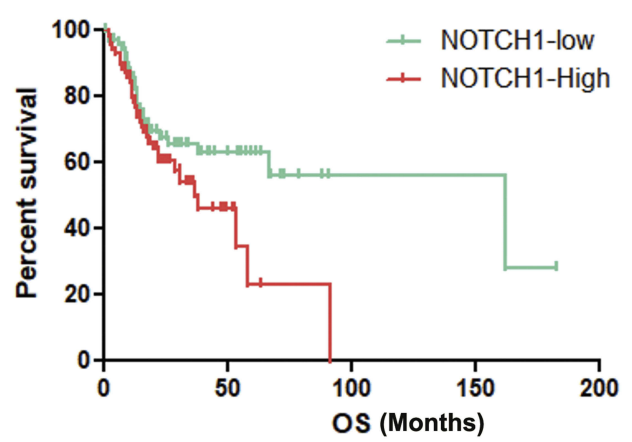

Figure I High expression of Notchl is associated with a poor prognosis in tongue cancer patients. (A) The TCGA database showed that the mRNA expression level of Notch I in tongue cancer was not different from that in normal tissues; TC represents tongue cancer, and $\mathrm{N}$ represents normal tissues. (B) The rate of overall survival in the high Notch I group was lower than that of the low Notch I group. 
A

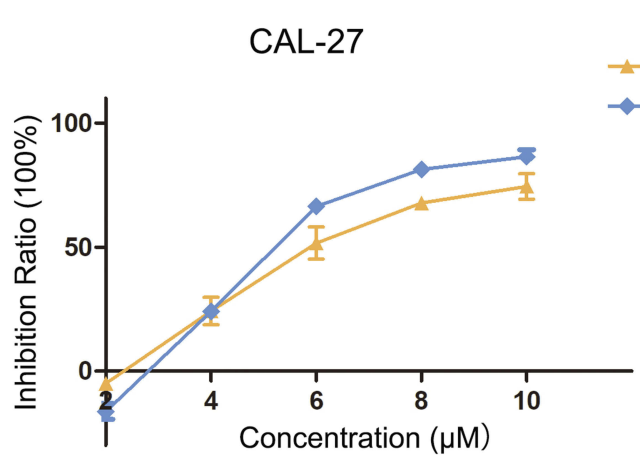

C

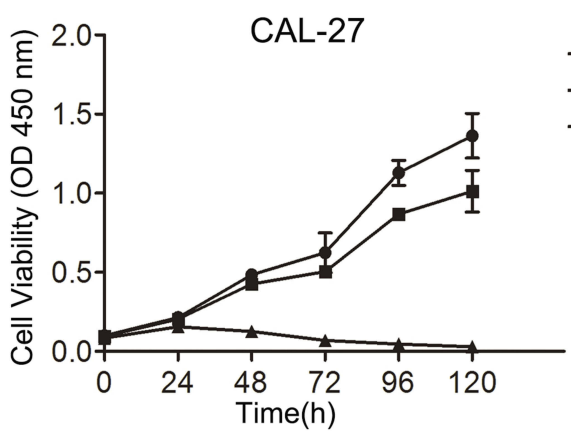

$\mathrm{E}$

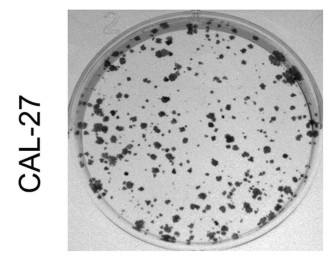

FLI-06 0 $\mathrm{MM}$

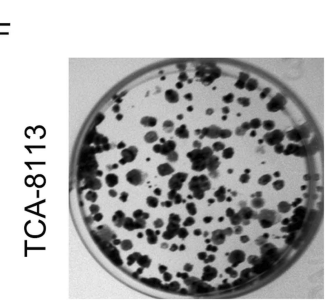

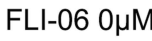

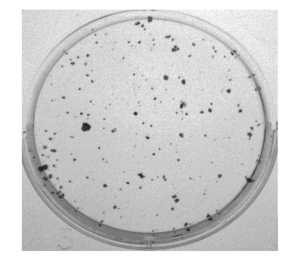

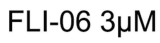

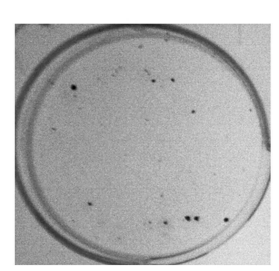

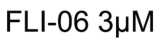

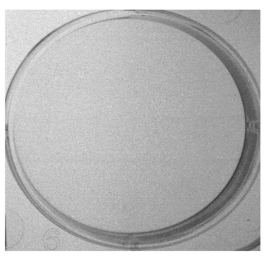

FLI-06 10 $\mu \mathrm{M}$

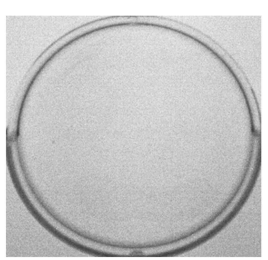

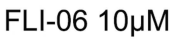

B

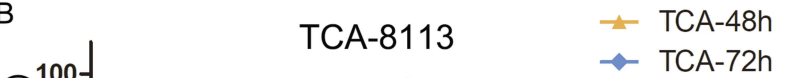

- CAL-48h

CAL-72h

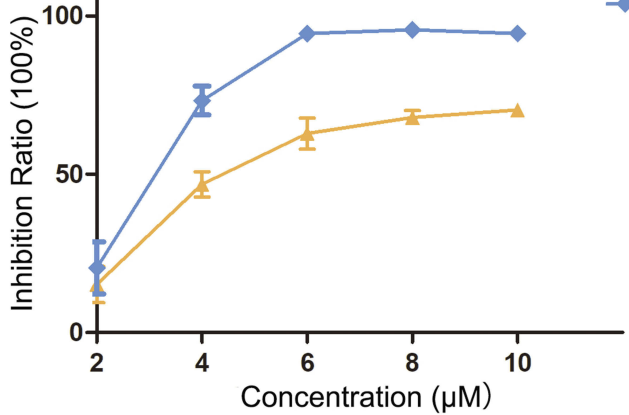

D
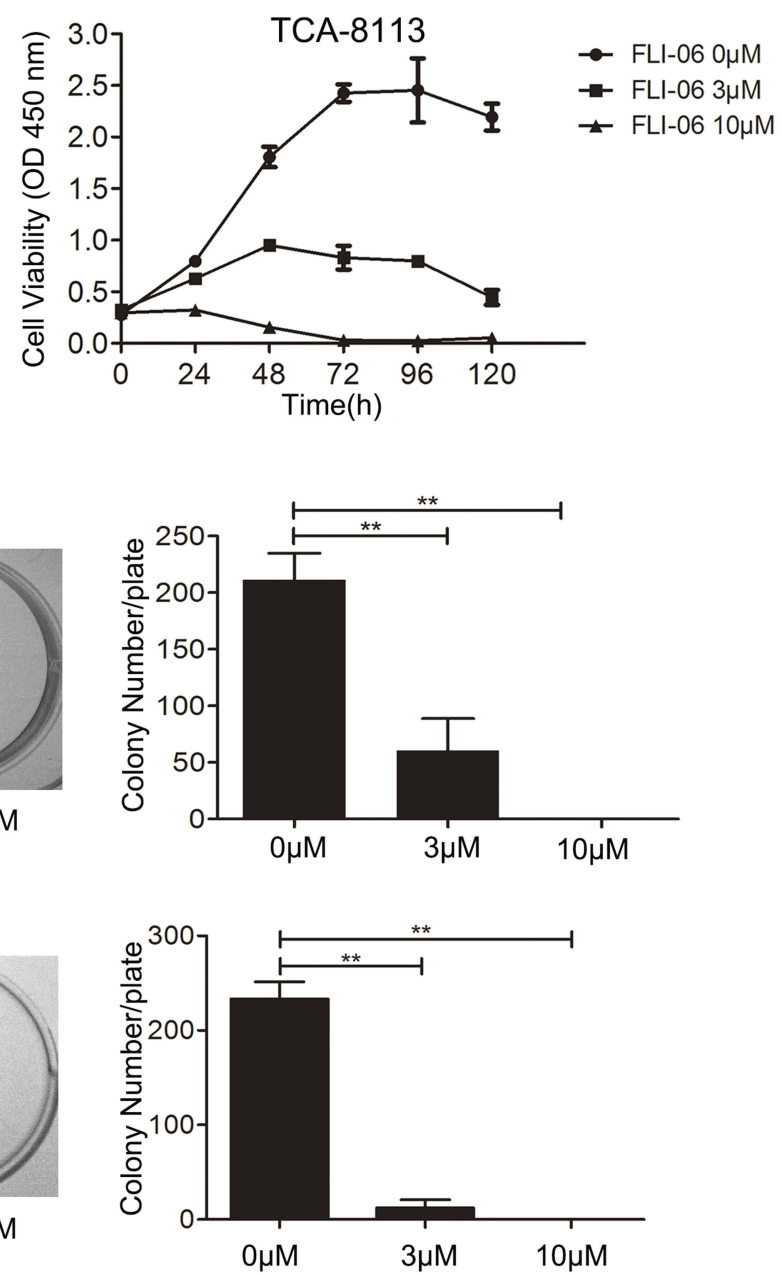

Figure 2 Blocking Notch I with FLI-06 inhibited tumor growth in vitro. (A, B) After treatment with FLI-06 at different concentrations for $48 \mathrm{~h}$ or $72 \mathrm{~h}$, the viability of CAL27 (A) and TCA-8II3 (B) cells was measured by the CCK8 assay; CAL-27 and TCA-8I I 3 cells proliferation ability was measured by the CCK8 (C, D) and colony formation (E, F) assays. Representative photomicrographs of the plates are shown in the left panel, and the colony number of each plate is shown in the right panel. Data represent the mean \pm S.D. of three independent experiments $(* *$ indicates $P<0.01)$.

\section{Notch Signaling Pathway Inhibitor Promotes Tongue Cancer Cell Apoptosis And Arrest The Cell Cycle}

Since FLI-06 can inhibit the proliferation of CAL-27 cells, we further investigated whether this inhibition of proliferation is associated with an increase in cell apoptosis or cell cycle arrest. As expected, annexin V-PI staining flow cytometry showed that the percentage of cells in early and late apoptosis increased after treatment with FLI-06 (Figure 3A). At the same time, we verified cell apoptosis using TUNEL assays. The results demonstrated that the number of CAL-27 cells 
A

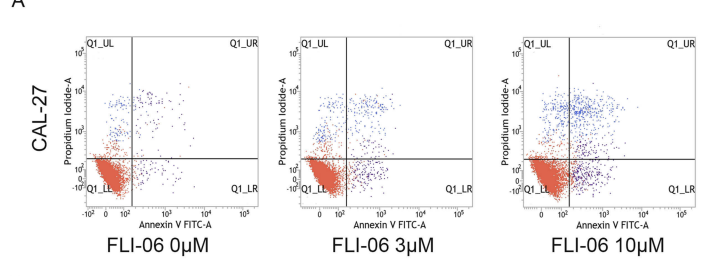

B
FLI-06 0 $\mu \mathrm{M}$

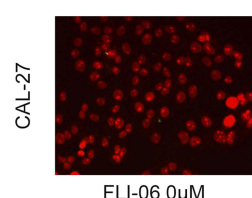

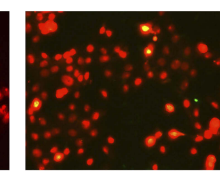

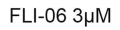

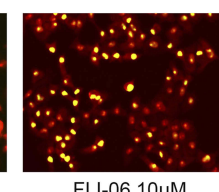

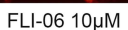
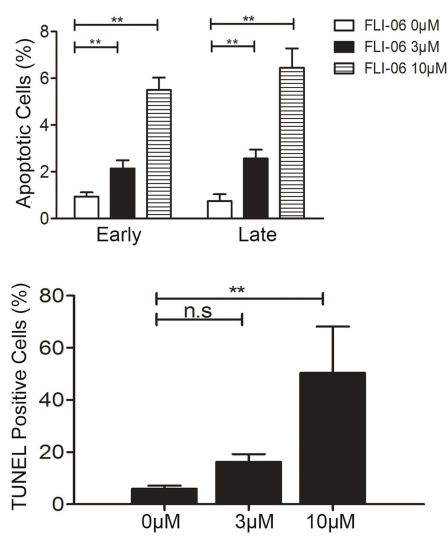

C
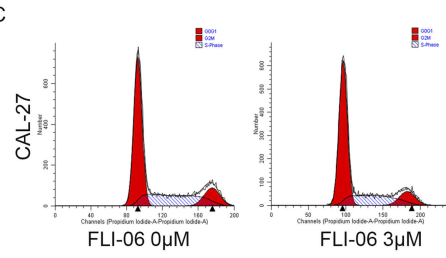

:

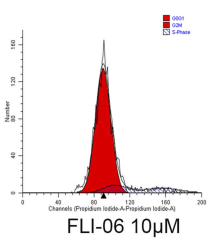

则:

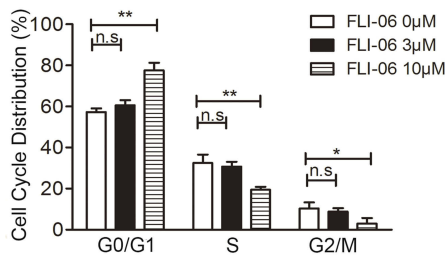

D
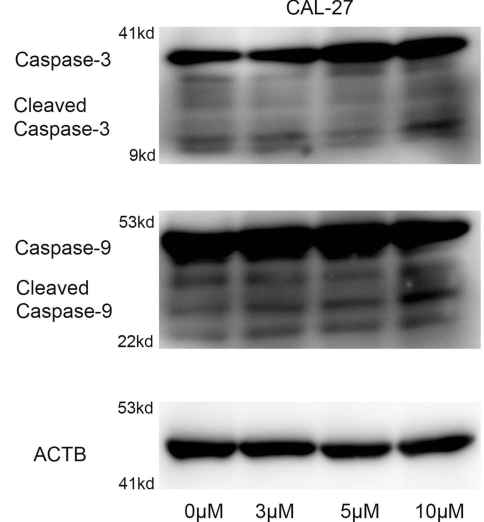

Figure 3 FLI-06 induced apoptosis and arrested the cell cycle in CAL-27 cells. (A) Annexin V-PI staining flow cytometry showed that the number of early and late apoptotic cells increased following FLI-06 treatment; (B) The TUNEL assay showed that in the negative control group, TUNEL-positive cells were fewer than that in the FLI-06 treatment groups; (C) The PI staining flow cytometry cycle test demonstrated that in the high concentration group (I0 $\mu$ M FLI-06), the cells were restrained in G0/GI phase. (D) The expression of the apoptosis-related genes Caspase-3 and Caspase-9 was measured by Western blotting in CAL-27 cells with or without FLI-06 treatment. Data represent the mean \pm S.D. of two independent experiments (* indicates $P<0.05$ and $* *$ indicates $P<0.01$ ).

Abbreviation: n.s, not significant.

with yellow fluorescence, which represents TUNEL-positive or apoptotic cells, in the treated groups was greater than that in the control group (Figure 3B). In other words, the number of apoptotic cells in the treatment groups increased. We also utilized Western blotting to detect the protein expression of Caspase-3 and Caspase-9. From the data (Figure 3D) we concluded that the cleaved Caspase- 3 and cleaved Caspase-9 protein levels were elevated in the FLI-06 groups compared with group without FLI-06 treatment. To test whether the increase in cell apoptosis was related to cell cycle arrest, we performed PI staining flow cytometry. The results demonstrated that the cell cycle of CAL-27 cells treated with $10 \mu \mathrm{M}$ FLI-06 was blocked in G0/G1 phase (Figure 3C). The proper concentration of FLI-06 treatment in CAL-27 cells increased cell apoptosis and blocked cell cycle arrest.

\section{FLI-06 Restrains Tumor Growth In The Xenograft Cancer Model}

Since we achieved relatively good results in vitro, we further studied the impact of FLI-06 on tumor growth in vivo. The xenograft cancer nude mouse model was successfully established. Four days after the injection of CAL-27 cells, a daily intraperitoneal injection of FLI-06 at a dose of $40 \mathrm{mg} / \mathrm{kg}$ body weight for nude mice was given, with administration for 6 days and withdrawal for 2 days, and treatment was stopped 2 cycles later. The mice were sacrificed 2 days after drug withdrawal, and the tumors were removed. The normal saline and DMSO control groups were established, and body weight and tumor volume were measured every other day. The results indicated that the body weights (Figure 4A) of the mice showed no variation, while the tumor volume (Figure 4B) and tumor weight (Figure 4C and D) of the FLI-06 treatment group were decreased compared with the control group. After treatment with FLI-06, the expression of Ki67 decreased, but the expression of Caspase-9 increased (Figure 4E and F) in xenograft tumors.

\section{FLI-06 Could Suppress The Protein Expression Of Notch Receptor}

We studied the effect of FLI-06 on the activation of the Notch signaling pathway. After $48 \mathrm{~h}$ of treatment with FLI-06, the mRNA expression of NOTCH1, NOTCH2, HEY1, HES1 and HEY2 was downregulated (Figure 5A), as measured by real-time PCR, and the protein levels of Notch1 full length, NICD-1 and downstream proteins HES1 and HEY2 were decreased in CAL-27 cells, as shown by Western blotting (Figure 5B and C). These 

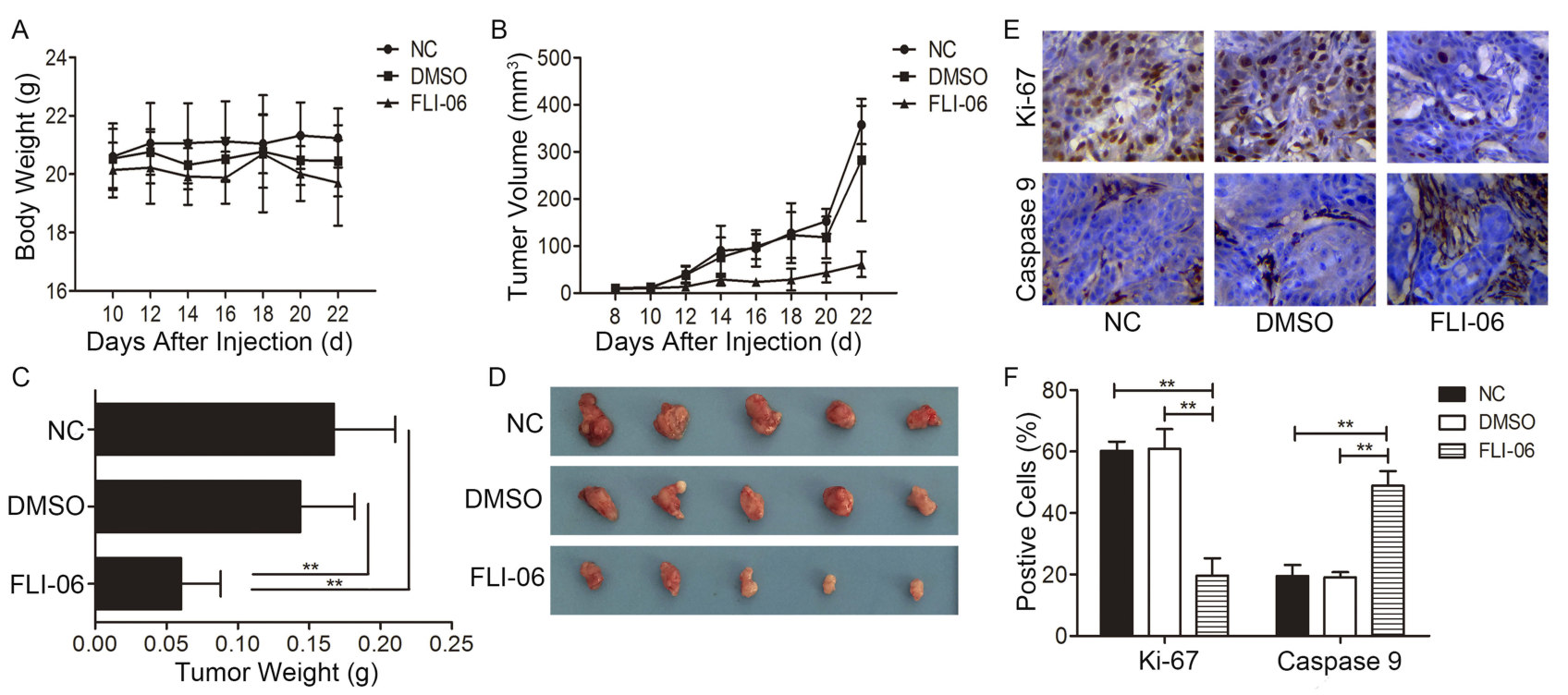

Figure 4 FLI-06 inhibited tumor growth in vivo. The changes in body weight $(\mathbf{A})$ and tumor volume (B) of nude mice were monitored during the experiment, and this graph shows no significant change in body weight among the groups, while the tumor volume of the FLI-06-treated group was smaller than that of the control; (C, D) The animals were sacrificed, and the tumors were removed for weighing $(\mathbf{C})$ and photographing (D) The column graph shows that the tumor weight of the FLI-06 treatment group was less than that of the control group, and the photograph of the tumors shows a similar trend; (E, F) The expression of Ki-67 (upper panel) and cleaved Caspase-9 (lower panel) in xenograft tumors using immunohistochemistry (DAB, 400x) $(* *$ indicates $P<0.01)$.

results prove that FLI-06 could suppress the protein expression of Notch receptor and block the Notch signaling pathway activation.

\section{The Self-Renewal Ability Of Tongue Cancer Stem Cells Is Inhibited By FLI-06}

To explore the influence of FLI-06 on tongue cancer stem cells, we performed a cancer stem cell sphere formation assay. CAL-27 or TCA-8113 tongue cancer stem cells were treated with FLI-06 for 15 days or 5 days, respectively. The proliferation of tongue cancer stem cells was notably inhibited by FLI-06 in the $3 \mu \mathrm{M}$ or $2 \mu \mathrm{M}$ group (Figure 6A). In addition, we detected the expression levels of cancer stem cell biomarkers. After CAL-27 cells were treated with FLI-06 for $48 \mathrm{~h}$, the real-time PCR data showed that the mRNA expression levels of ALDH1, SOX2 and OCT4 were downregulated in the $3 \mu \mathrm{M}$ and 5 $\mu \mathrm{M}$ groups, while the expression level of SLUG was not altered (Figure 6B). In TCA-8113 cells, we found a decrease in ALDH1, SOX2 and SLUG expression in the FLI-06 groups, but the expression level of OCT4 among the groups was not significant (Figure 6B). To further detect the ability of FLI-06 to permeate cancer stem cell spheres, we treated cancer stem cells with the inhibitor after cancer stem cell sphere formation. From the data, we know that FLI-06 could permeate into and break the tongue cancer stem cell spheres (Figure 7). In CAL-27 cells, the stem cell spheres were destroyed after treatment with $3 \mu \mathrm{M}$ FLI-06 for 10 days (Figure 7A), and in TCA8113 cells, the stem cell spheres were destroyed after treatment with $2 \mu \mathrm{M}$ FLI-06 for 6 days (Figure 7B).

In vivo and in vitro experiments verified that FLI-06 could inhibit the growth of tongue cancer cells and be proapoptotic. The activity of the Notch signaling pathway was blocked by using FLI-06. Additionally, the proliferation ability of tongue cancer stem cells was inhibited by FLI-06. Therefore, our data provide an experimental basis for the clinical application of FLI-06 targeted therapy for tongue cancer.

\section{Discussion}

Tongue cancer is the most common oral cancer. A previous report stated that the incidence of tongue cancer is increasing, especially in the young population, in both developing and developed countries. ${ }^{2,23,24}$ For this reason, it is becoming increasingly crucial to find the molecular mechanism of tongue cancer and discover a new targeted treatment approach for patients. The abnormal activation of the Notch signaling pathway is related to many diseases, including cancer.

The function of the Notch signaling pathway in squamous cell carcinoma (SCC) remains a cause of much debate. Notch1 has been reported to be associated with 
A
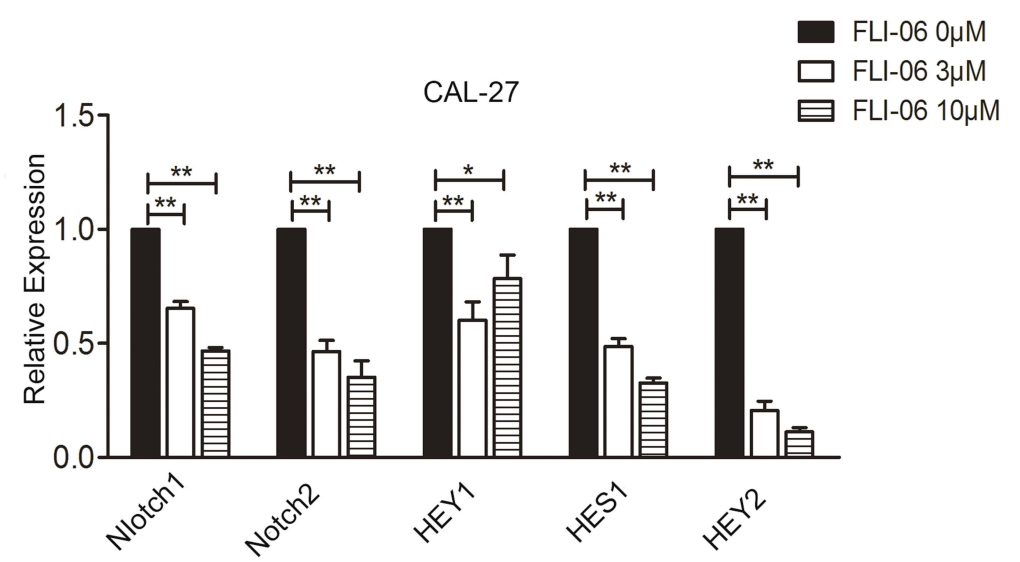

B
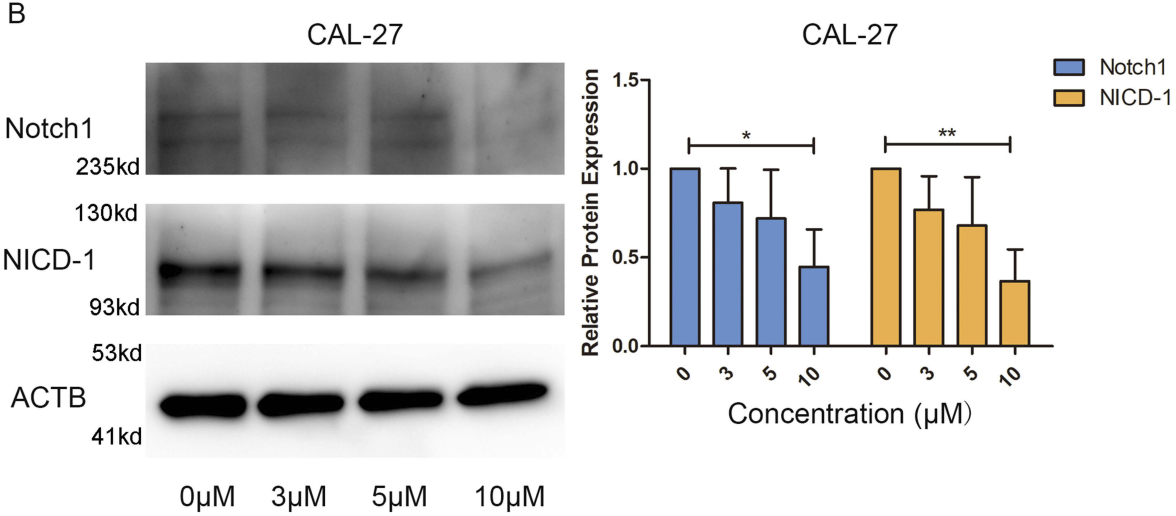

C
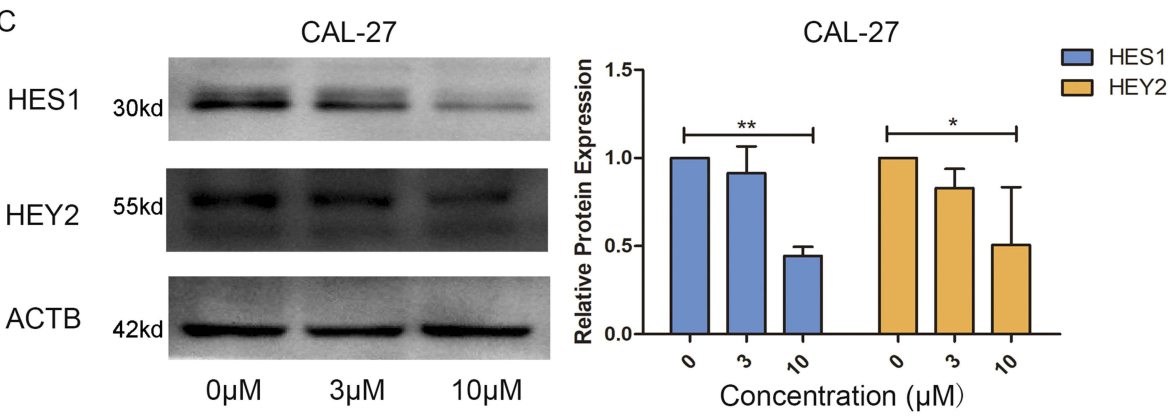

Figure 5 The protein expression of Notch and the activating of the pathway were inhibited by FLI-06. (A) Real-time PCR analysis showed lower expression of Notch target gene mRNA in the FLI-06 treatment group relative to the group without FLI-06 treatment; (B) Western blot analysis showed that the expression of both the full length of Notch I receptor and NICDI proteins in the FLI-06 treatment group was decreased compared to that of the control; (C) The protein expression level of HESI and HEY2, which are Notch signaling pathway targeted genes, were downregulated after treatment with FLI-06. Data represent the mean \pm S.D. of three independent experiments (* indicates $\mathrm{P}<0.05$ and $* *$ indicates $\mathrm{P}<0.01$ ).

tumor invasion in esophageal SCC and is related to a poor prognosis. $^{25,26}$ Fukusumi $^{27}$ suggested that the Notch4HEY1 pathway is upregulated in HNSCC and the overexpression of Notch1 is involved in HNSCC cancer cell proliferation and cisplatin resistance. Kayamori and colleagues $^{28}$ argued that the expression of Notch3 in cancer-associated fibroblasts is related to oral squamous cell carcinoma and promotes tumor angiogenesis. However, many studies have reported a tumor suppressor role for
Notch. For example, loss of function of the canonical Notch signaling pathway in HNSCC promotes tumorigenesis. $^{29}$ Wang and colleagues ${ }^{29}$ stated that lossof-function mutations in Notch are highly significant in lung and skin SCC patients. The cancer microenvironment, context and type of Notch mutation may lead to various roles for Notch in different cancers. ${ }^{30}$ Previous studies on the effect of Notch1 in tongue squamous cell carcinoma have emphasized the carcinogenic role of 

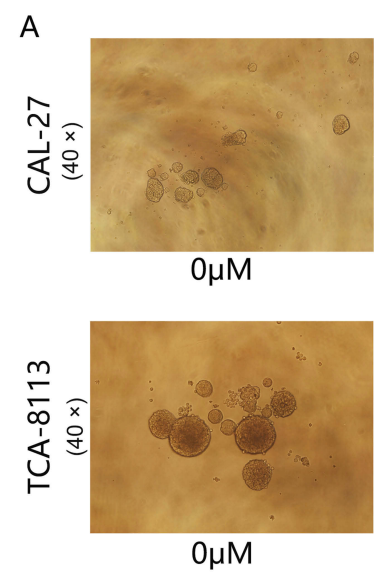

B

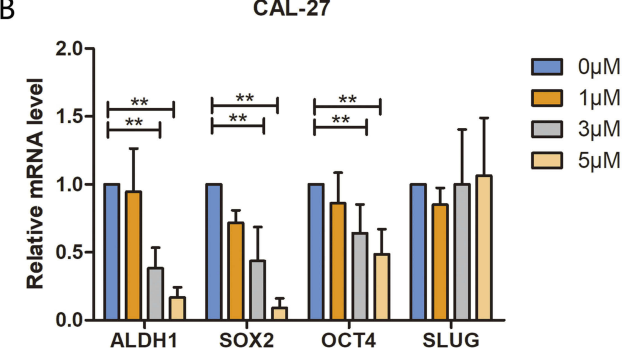

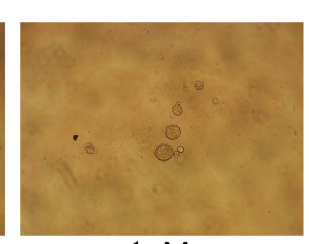

$1 \mu \mathrm{M}$

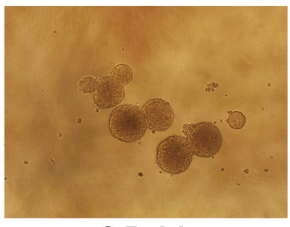

$0.5 \mu \mathrm{M}$

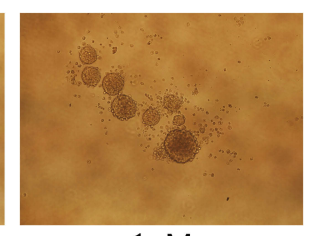

$1 \mu \mathrm{M}$

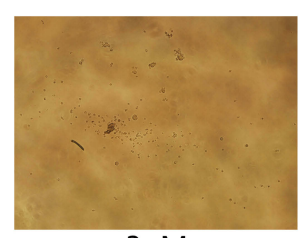

$3 \mu \mathrm{M}$

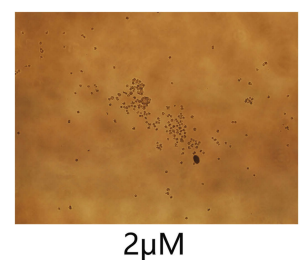

$2 \mu \mathrm{M}$
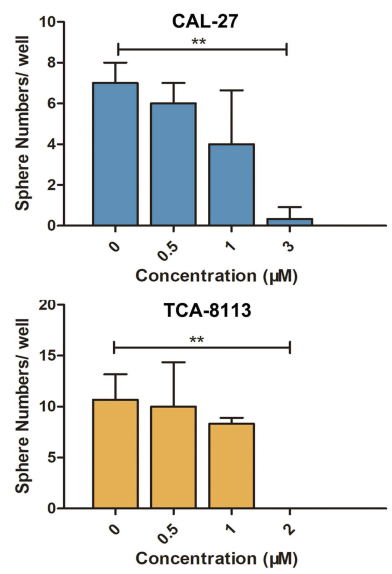

TCA-8113

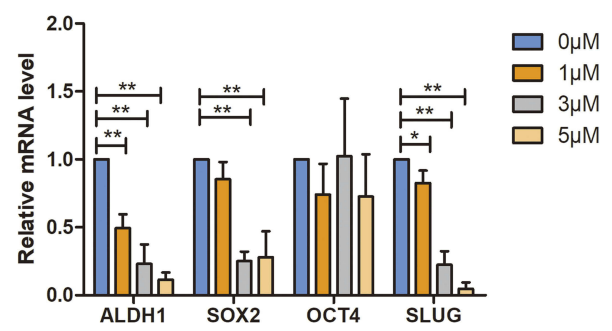

Figure 6 FLI-06 could inhibit the proliferation of tongue cancer stem cells. (A) FLI-06 was added to CAL-27 cancer stem cells (upper panel) for 10 days and TCA-8II3 cancer stem cells for 6 days (lower panel). FLI-06 inhibited the proliferation of stem cells in the high concentration group. Representative images are shown in the left panel (40x), and the statistical column is shown in the right panel. (B) The real-time PCR data demonstrated that the stem cell biomarkers were decreased in the FLI-06 treatment groups. Data represent the mean \pm S.D. of three independent experiments (* indicates $P<0.05$ and $* *$ indicates $P<0.01$ ).

Notch1 in tongue cancer. ${ }^{17}$ Therefore, we hope to find a suitable inhibitor to inhibit the activity of the Notch signaling pathway to treat tongue cancer.

Gamma secretase plays a key role in the Notch signaling pathway, and various studies have focused on applying GSIs (Gamma secretase inhibitors) to treat various types of cancer. Many studies have reported utilizing different kinds of GSIs to treat solid tumors, such as breast tumors, ${ }^{31}$ lung cancer, ${ }^{32}$ and esophageal adenocarcinoma, ${ }^{33}$ and most suppress the progression of tumor growth. There are many effective GSIs used in clinical experiments (https://www.clinical trials.gov). When used in HNSCC, GSIs reduced the number of immunosuppressive cells. ${ }^{34}$ Even though a gastrointestinal reaction following GSI treatment has been observed, the use of GSIs as a new approach to targeting the Notch pathway is promising. There are few reports on the use of FLI-06 in solid tumors in an animal experiment. FLI-06 inhibited the proliferation and induced the apoptosis of tongue cancer cells in vitro. On the basis of our real-time PCR and Western blot data, we found that growth suppression was related with inhibition of the Notch signaling pathway, and we examined whether the expression of NICD-1, NICD-2, HES1 and HEY2 was downregulated after FLI-06-treatment in CAL27 cells. Meanwhile, Ki-67 is a highly acknowledged proliferation gene, and Caspase-9 is a mature apoptotic gene. We found that the expression of $\mathrm{Ki}-67$ was decreased while Caspase-9 expression was increased in the transplant tumors of the treatment groups. Cancer cells are always in caryomitosis, which is divided into four phases, and if mitotic cells are blocked in any phase, apoptosis occurs. Cell cycle arrest is a key to treating cancer. ${ }^{35}$ Therefore, we studied the cell cycle of tongue cancer cells, and our data indicate that the increased rate of apoptosis is related to cell cycle arrest. The cell cycle was blocked in G0/G1 phase after FLI-06 treatment, consistent with the effects of GSIs on other solid tumors, which also exhibit cell cycle arrest. ${ }^{36,37}$ Cancer stem cells may be related to the malignant characteristics of cancer, such as metastasis and recurrence. ${ }^{38,39}$ It is meaningful to decrease or eliminate the population of cancer stem cells. The Notch signaling pathway plays an important role in cancer stem cells. ${ }^{40}$ Adenoid cystic carcinoma stem cells have the feature of activated Notch1, and GSIs could deplete 
A
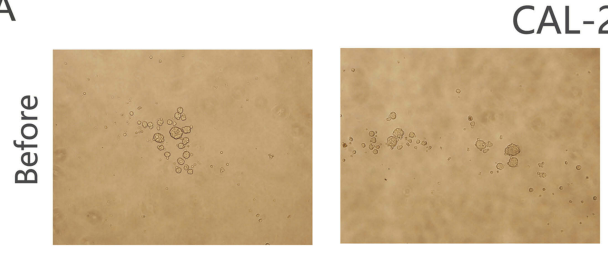

$(40 \times)$

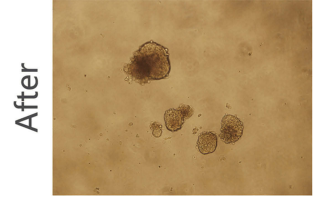

$0 \mu \mathrm{M}$

B
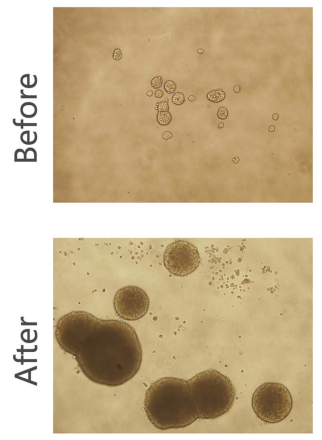

$0 \mu \mathrm{M}$

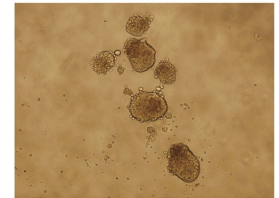

$0.5 \mu \mathrm{M}$

TCA-8113 (40x)
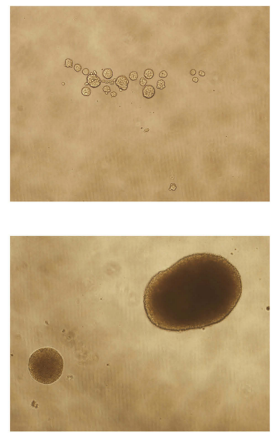

$0.5 \mu \mathrm{M}$
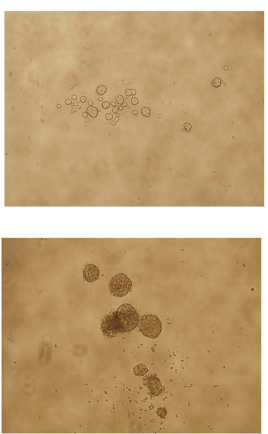

$1 \mu \mathrm{M}$
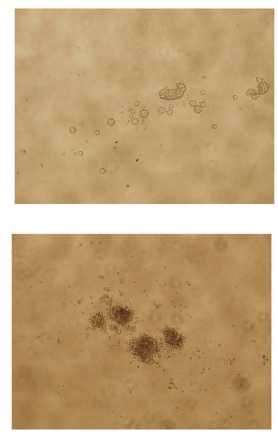

$3 \mu \mathrm{M}$
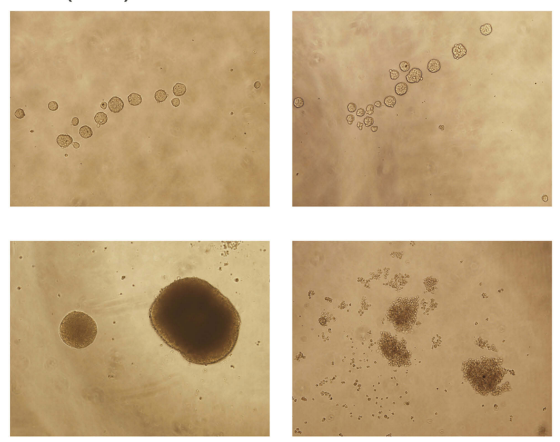

$1 \mu \mathrm{M}$

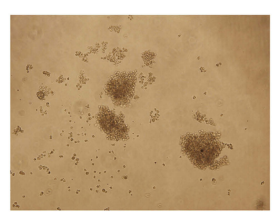

$2 \mu \mathrm{M}$

Figure 7 FLI-06 could block the self-renewal ability of tongue cancer stem cells. FLI-06 was used to treat tongue cancer stem cell spheres. (A) After CAL-27 cancer stem cell spheres were treated with FLI-06 for 8 days, the spheres were broken in the $3 \mu \mathrm{M}$ concentration group (40x). (B) After TCA-8II3 cancer stem cell spheres were treated with FLI-06 for 4 days, the spheres were completely broken in the $2 \mu \mathrm{M}$ concentration group (40x).

the numbers of cancer stem cells. ${ }^{41} \mathrm{Ma}^{42}$ demonstrated that Notch3 facilitated stem cell capacity in lung cancer.

\section{Conclusions}

Our study showed that FLI-06 could block Notch activation and then decrease the self-renewal ability of tongue cancer stem cells. These results illustrate an effective FLI06 that most likely has promising antitumoral activity and can be used as a targeted chemotherapeutic agent for the clinical treatment of tongue tumors.

\section{Ethics approval and consent to participate}

All animal experiments were performed according to the guidelines for the care and use of laboratory animals and the protocol was approved by the biomedical ethics research committee of Fujian Medical University (2016-34).

\section{Abbreviations}

GSI, Gamma secretase inhibitor; ATCC, American Type Culture Collection; CCK8, Cell Counting Kit-8; $\mathrm{IC}_{50}$, the half maximal inhibitory concentration.

\section{Acknowledgments}

This work was supported by grants from the National Natural Sciences Foundation of China (grant numbers 81641105 and 81872186), Natural Sciences Foundation of Fujian Province (grant number 2017J01520), Scientific Research Funding of School and Hospital of Stomatology, Fujian Medical University (grant number 2018KQYJ01). The authors thank Dr. Wantao Chen (Department of Oral and Maxillofacial-Head and Neck Oncology, Ninth People's Hospital, Shanghai Jiao Tong University School of Medicine, Shanghai, China) for kindly providing the tongue cancer cell line TCA-8113. 


\section{Author contributions}

YL, DZ, RG presented the study concepts. YL, DZ, RG, XP designed this study. RG, LL, JX, LH, LD, BS in charge of data acquisition. RG, LL, LH, LD, BS controlled the quality of data and algorithms. RG, DZ, YL, XP analyzed and interpreted the data. XP, LD, BS performed the statistical analysis. RG, JX, DZ had a major contributor in writing the manuscript. All authors contributed to data analysis, drafting and revising the article, gave final approval of the version to be published, and agree to be accountable for all aspects of the work.

\section{Data sharing statement}

All data generated or analysed during this study are included in this published article and are available from the corresponding author on reasonable request.

\section{Disclosure}

The authors report no conflicts of interest in this work.

\section{References}

1. Zhang LW, Li J, Cong X, et al. Incidence and mortality trends in oral and oropharyngeal cancers in China, 2005-2013. Cancer Epidemiol. 2018;57:120-126. doi:10.1016/j.canep.2018.10.014

2. Hussein AA, Helder MN, de Visscher JG, et al. Global incidence of oral and oropharynx cancer in patients younger than 45 years versus older patients: a systematic review. Eur J Cancer. 2017;82:115-127. doi:10.1016/j.ejca.2017.05.026

3. Ng JH, Iyer NG, Tan MH, Edgren G. Changing epidemiology of oral squamous cell carcinoma of the tongue: a global study. Head Neck. 2017;39(2):297-304. doi:10.1002/hed.24589

4. Dexter JS. The analysis of a case of continuous variation in drosophila by a study of its linkage relations. Am Nat. 1914;48(576):712758. doi: $10.1086 / 279446$

5. Siebel C, Lendahl U. Notch signaling in development, tissue homeostasis, and disease. Physiol Rev. 2017;97(4):1235-1294. doi:10.1152/physrev.00005.2017

6. Kopan R, Ilagan MX. The canonical Notch signaling pathway: unfolding the activation mechanism. Cell. 2009;137(2):216-233. doi:10.1016/j.cell.2009.03.045

7. Zanotti S, Canalis E. Notch signaling and the skeleton. Endocr Rev. 2016;37(3):223-253. doi:10.1210/er.2016-1002

8. Fortini ME. Gamma-secretase-mediated proteolysis in cell-surface-receptor signalling. Nat Rev Mol Cell Biol. 2002;3(9):673-684. doi:10.1038/ nrm910

9. Takebe N, Nguyen D, Yang SX. Targeting Notch signaling pathway in cancer: clinical development advances and challenges. Pharmacol Ther. 2014;141(2):140-149. doi:10.1016/j.pharmthera. 2013.09.005

10. Kovall RA, Gebelein B, Sprinzak D, Kopan R. The canonical Notch signaling pathway: structural and biochemical insights into shape, sugar, and force. Dev Cell. 2017;41(3):228-241. doi:10.1016/j. devcel.2017.04.001

11. Sanchez-Martin M, Ferrando A. The NOTCH1-MYC highway toward T-cell acute lymphoblastic leukemia. Blood. 2017;129 (9):1124-1133. doi:10.1182/blood-2016-09-692582
12. Hayashi T, Gust KM, Wyatt AW, et al. Not all NOTCH is created equal: the oncogenic role of NOTCH2 in bladder cancer and its implications for targeted therapy. Clin Cancer Res. 2016;22 (12):2981-2992. doi:10.1158/1078-0432.CCR-15-2360

13. Zhang J, Kuang Y, Wang Y, Xu Q, Ren Q. Notch-4 silencing inhibits prostate cancer growth and EMT via the NF-kappaB pathway. Apoptosis. 2017;22(6):877-884. doi:10.1007/s10495-017-1368-0

14. Giachino C, Boulay JL, Ivanek R, et al. A tumor suppressor function for Notch signaling in forebrain tumor subtypes. Cancer Cell. 2015;28(6):730-742. doi:10.1016/j.ccell.2015.10.008

15. South AP, Purdie KJ, Watt SA, et al. NOTCH1 mutations occur early during cutaneous squamous cell carcinogenesis. J Invest Dermatol. 2014;134(10):2630-2638. doi:10.1038/jid.2014.154

16. Wang WJ, Yao Y, Jiang LL, et al. Increased LEF1 expression and decreased Notch2 expression are strong predictors of poor outcomes in colorectal cancer patients. Dis Markers. 2013;35(5):395-405. doi:10.1155/2013/983981

17. Gan RH, Wei H, Xie J, et al. Notch1 regulates tongue cancer cells proliferation, apoptosis and invasion. Cell Cycle. 2018;17(2):216224. doi:10.1080/15384101.2017.1395534

18. Gomez-Galeno JE, Hurtado C, Cheng J, Yardimci C, Mercola M, Cashman JR. b-annulated 1,4-dihydropyridines as Notch inhibitors. Bioorg Med Chem Lett. 2018;28(20):3363-3367. doi:10.1016/j. bmcl.2018.09.002

19. Yonemura YLX, Müller K, Krämer A, et al. Inhibition of cargo export at ER-exit sites and the trans-golgi network by the secretion inhibitor FLI06. J Cell Sci. 2016;129(20):3868-3877. doi:10.1242/jcs. 186163

20. Kramer A, Mentrup T, Kleizen B, et al. Small molecules intercept Notch signaling and the early secretory pathway. Nat Chem Biol. 2013;9(11):731-738. doi:10.1038/nchembio. 1356

21. Lu Z, Ren Y, Zhang M, et al. FLI-06 suppresses proliferation, induces apoptosis and cell cycle arrest by targeting LSD1 and Notch pathway in esophageal squamous cell carcinoma cells. Biomed Pharmacother. 2018;107:1370-1376. doi:10.1016/j.biopha.2018.08.140

22. Qu J, Song M, Xie J, et al. Notch2 signaling contributes to cell growth, invasion, and migration in salivary adenoid cystic carcinoma. Mol Cell Biochem. 2016;411(1-2):135-141. doi:10.1007/s11010015-2575-Z

23. Ghantous Y, Abu Elnaaj I. [Global incidence and risk factors of oral cancer]. Harefuah. 2017;156(10):645-649.

24. Tota JE, Anderson WF, Coffey C, et al. Rising incidence of oral tongue cancer among white men and women in the United States, 1973-2012. Oral Oncol. 2017;67:146-152. doi:10.1016/j. oraloncology.2017.02.019

25. Lubin DJ, Mick R, Shroff SG, Stashek K, Furth EE. The Notch pathway is activated in neoplastic progression in esophageal squamous cell carcinoma. Hum Pathol. 2018;72:66-70. doi:10.1016/j. humpath.2017.11.004

26. Natsuizaka M, Whelan KA, Kagawa S, et al. Interplay between Notch1 and Notch3 promotes EMT and tumor initiation in squamous cell carcinoma. Nat Commun. 2017;8(1):1758. doi:10.1038/s41467017-01500-9

27. Fukusumi T, Guo TW, Sakai A, et al. TheNOTCH4-HEY1Pathway induces epithelial-mesenchymal transition in head and neck squamous cell carcinoma. Clin Cancer Res. 2018;24(3):619-633. doi:10.1158/1078-0432.CCR-17-1366

28. Kayamori K, Katsube K, Sakamoto K, et al. NOTCH3 is induced in cancer-associated fibroblasts and promotes angiogenesis in oral squamous cell carcinoma. PLoS One. 2016;11(4):e0154112. doi:10.1371/ journal.pone.0154112

29. Nyman PE, Buehler D, Lambert PF. Loss of function of canonical Notch signaling drives head and neck carcinogenesis. Clin Cancer Res. 2018;24(24):6308-6318. doi:10.1158/1078-0432.CCR-17-3535

30. Aster JC, Pear WS, Blacklow SC. The varied roles of Notch in cancer. Annu Rev Pathol. 2017;12:245-275. doi:10.1146/annurevpathol-052016-100127 
31. Schott AF, Landis MD, Dontu G, et al. Preclinical and clinical studies of gamma secretase inhibitors with docetaxel on human breast tumors. Clin Cancer Res. 2013;19(6):1512-1524. doi:10.1158/10780432.CCR-11-3326

32. Osanyingbemi-Obidi J, Dobromilskaya I, Illei PB, Hann CL, Rudin CM. Notch signaling contributes to lung cancer clonogenic capacity in vitro but may be circumvented in tumorigenesis in vivo. Mol Cancer Res. 2011;9(12):1746-1754. doi:10.1158/1541-7786.MCR-11-0286

33. Wang Z, Da Silva TG, Jin K, et al. Notch signaling drives stemness and tumorigenicity of esophageal adenocarcinoma. Cancer Res. 2014;74(21):6364-6374. doi:10.1158/0008-5472.CAN-14-2051

34. Mao L, Zhao ZL, Yu GT, et al. gamma-secretase inhibitor reduces immunosuppressive cells and enhances tumour immunity in head and neck squamous cell carcinoma. Int J Cancer. 2018;142(5):999-1009. doi:10.1002/ijc.31115

35. Lin R, Zhang Z, Chen L, et al. Dihydroartemisinin (DHA) induces ferroptosis and causes cell cycle arrest in head and neck carcinoma cells. Cancer Lett. 2016;381(1):165-175. doi:10.1016/j.canlet.2016.07.033

36. Hu J, Zhu X, Lu Q. Antiproliferative effects of gamma-secretase inhibitor, a Notch signalling inhibitor, in multiple myeloma cells and its molecular mechanism of action. J Int Med Res. 2013;41 (4):1017-1026. doi:10.1177/0300060513485912
37. Liu X, Xu QR, Xie WF, Wang MD. DAPT suppresses the proliferation of human glioma cell line SHG-44. Asian Pac J Trop Med. 2014;7(7):552-556. doi:10.1016/S1995-7645(14)60092-4

38. Dawood S, Austin L, Cristofanilli M. Cancer stem cells: implications for cancer therapy. Oncology (Williston Park). 2014;28(12):11011107,1110

39. Ishiguro T, Ohata H, Sato A, Yamawaki K, Enomoto T, Okamoto K. Tumor-derived spheroids: relevance to cancer stem cells and clinical applications. Cancer Sci. 2017;108(3):283-289. doi:10.1111/ cas. 13155

40. Takebe N, Miele L, Harris PJ, et al. Targeting Notch, hedgehog, and Wnt pathways in cancer stem cells: clinical update. Nat Rev Clin Oncol. 2015;12(8):445-464. doi:10.1038/nrclinonc.2015.61

41. Panaccione A, Chang MT, Carbone BE, et al. NOTCH1 and SOX10 are essential for proliferation and radiation resistance of cancer stemlike cells in adenoid cystic carcinoma. Clin Cancer Res. 2016;22 (8):2083-2095. doi:10.1158/1078-0432.CCR-15-2208

42. Ma Y, Li M, Si J, et al. Blockade of Notch3 inhibits the stem-like property and is associated with ALDH1A1 and CD44 via autophagy in non-small lung cancer. Int $J$ Oncol. 2016;48(6):2349-2358. doi:10.3892/ijo.2016.3464

\section{Publish your work in this journal}

OncoTargets and Therapy is an international, peer-reviewed, open access journal focusing on the pathological basis of all cancers, potential targets for therapy and treatment protocols employed to improve the management of cancer patients. The journal also focuses on the impact of management programs and new therapeutic agents and protocols on patient perspectives such as quality of life, adherence and satisfaction. The manuscript management system is completely online and includes a very quick and fair peer-review system, which is all easy to use. Visit http://www.dovepress.com/ testimonials.php to read real quotes from published authors. 